\title{
Kernos
}

Revue internationale et pluridisciplinaire de religion grecque antique

$27 \mid 2014$

Varia

\section{Richard BUXTON, Myths \& Tragedies in their Ancient Greek Contexts}

\section{Ajda Latifses}

\section{(2) OpenEdition \\ Journals}

\section{Édition électronique}

URL : http://journals.openedition.org/kernos/2233

DOI : $10.4000 /$ kernos.2233

ISSN : 2034-7871

\section{Éditeur}

Centre international d'étude de la religion grecque antique

\section{Édition imprimée}

Date de publication : 1 novembre 2014

Pagination : 450-455

ISBN : 978-2-87562-055-2

ISSN : 0776-3824

\section{Référence électronique}

Ajda Latifses, «Richard Buxton, Myths \& Tragedies in their Ancient Greek Contexts », Kernos [En ligne], 27| 2014, mis en ligne le 12 novembre 2014, consulté le 22 septembre 2020. URL : http:// journals.openedition.org/kernos/2233; DOI : https://doi.org/10.4000/kernos.2233

Ce document a été généré automatiquement le 22 septembre 2020.

Kernos 


\title{
Richard BUXTON, Myths \& Tragedies in their Ancient Greek Contexts
}

\author{
Ajda Latifses
}

\section{RÉFÉRENCE}

Richard BUXTON, Myths \& Tragedies in their Ancient Greek Contexts, Oxford, Oxford

University Press, 2013. 1 vol. 14,5 × 22 cm, xii+280 p. ISBN : 978-0-19-955761-5.

1 Dans la seconde moitié du $\mathrm{xx}^{\mathrm{e}}$ siècle, l'étude de la mythologie grecque a connu, sous l'influence du structuralisme lévi-straussien, un profond renouvellement théorique et méthodologique, qui n'est cependant pas allé sans désillusions ni remises en question. La plus violente d'entre elles fut sans doute portée par l'ouvrage de Marcel Detienne, L'invention de la mythologie, publié en 1981. L'auteur y dénonçait les errances du structuralisme alors dominant, en insistant sur l'impossibilité de substantialiser le mythe pour le réduire à un ensemble de relations et d'oppositions binaires immanentes sans prise en compte de son contexte d'énonciation. Les mythes, rappelait M. Detienne, n'existent que dans la diversité de leurs versions et des formes énonciatives (langagières ou iconographiques) dans lesquelles elles apparaissent. Dès lors l'interprète, plutôt que de rechercher d'hypothétiques structures communes, se devrait de mettre en lumière les singularités de chaque version et la fonction particulière qui lui est assignée dans un contexte donné, défini à la fois en termes culturels et spatiotemporels (contexte extra-discursif), et en termes formels et génériques (contexte intra-discursif). C'est là la voie suivie par certains chercheurs, qui ont préféré tourner le dos à un structuralisme désormais suspect pour lui substituer une approche de type pragmatique. Mais le structuralisme n'en a pas moins continué à influencer, d'une manière plus ou moins consciente et assumée, bon nombre de travaux jusqu'à l'époque présente.

2 Sur ce fond d'incertitude théorique, le dernier ouvrage de R. Buxton (R.B.) offre le remarquable intérêt de mettre la réflexion théorique au cœur du discours, et de 
proposer une mise au point méthodologique à la fois claire, prudente et équilibrée, illustrée à travers des études de cas circonstanciés. Si le livre rassemble onze études publiées indépendamment entre 1987 et 2010, son unité lui vient d'une cohérence d'approche que l'auteur lui-même s'applique à mettre en valeur dans l'introduction, puis dans chacun des chapeaux introductifs qui ouvrent et coordonnent les chapitres successifs. D'une manière plus explicite et articulée qu'il ne l'avait fait dans son précédent ouvrage, La Grèce de l'imaginaire ${ }^{1}$, R.B. affiche ici le souci de concilier une approche structuraliste dont il réaffirme la validité, avec une attention rigoureuse et exigeante au contexte des versions mythiques étudiées. C'est, disons-le d'emblée, ce discours de la méthode en acte qui constitue le principal intérêt de l'ouvrage; les conclusions auxquelles l'A. aboutit, quant à elles, demeurent souvent assez traditionnelles, mais ne s'en distinguent pas moins par leur sens de la nuance et de la circonspection.

Pour penser l'articulation et l'interaction entre le niveau de la structure et celui du contexte, l'A. recourt à une métaphore linguistique (particulièrement développée au chapitre 11) empruntée à la terminologie saussurienne - elle-même préstructuraliste - l'opposition entre « langue » et « parole ». À la suite de Claude Lévi-Strauss, il admet qu'une société puisse emprunter certains aspects du monde empirique environnant pour élaborer des paires d'oppositions binaires "bonnes à penser ». Ce système de représentations, qui conserve une certaine stabilité au cours de l'histoire grecque, constitue la «langue » du mythe, tandis que chacune de ses réalisations littéraires ou artistiques singulières apparaît comme une manifestation de la "parole ». La « langue » mythique, notons-le cependant d'emblée, ne se réduit jamais tout à fait chez R.B. au jeu schématique des oppositions binaires: dès l'introduction, il associe à l'approche rigoureusement structuraliste une approche thématique plus souple. Pour reconstituer au mieux la « langue » mythique, l'A. insiste en outre sur la fécondité méthodologique qu'offrent les rapprochements entre énoncés mythiques et énoncés considérés comme non mythiques (relevant par exemple du discours historique, médical ou botanique) : à la suite de L. Gernet, dont il cite l'exemple, R.B. affirme la « porosité » du mythe, qui ne saurait constituer un mode de pensée à part, dissociable d'une pensée prétendument rationnelle. Par ailleurs, afin de mieux comprendre comment se construisent les représentations mythiques, il souligne la nécessité de confronter les mythes aux realia de la société et de l'environnement grecs: ainsi intervient l'attention au contexte qui constitue l'autre élément-clef de la méthode de R.B.

4 Alors cependant que dans La Grèce de l'imaginaire, le contexte du mythe tendait à se réduire à la réalité du monde grec antique, R.B. affirme ici l'importance de prendre également en compte les moyens de la représentation mythique, c'est-à-dire les formes discursives et iconographiques dans lesquelles chaque "parole» mythique se déploie. L'attention portée au contexte doit prémunir contre deux écueils herméneutiques, d'une part le risque d'interpréter mécaniquement, en leur plaquant un sens tout fait, des récurrences structurales et thématiques en fait resémantisées dans chaque contexte singulier, de l'autre le mirage déconstructiviste d'une infinie ouverture du sens, en réalité toujours limité par les potentialités de la «langue » mythique comme par les choix que détermine le contexte. La prise en considération du genre, qui impose ses codes à la matière traitée et dicte un horizon d'attente au destinataire, remplit dans cette perspective un rôle majeur. Comme l'indique le titre même de l'ouvrage, Myths and Tragedies, c'est alors la tragédie attique qui fournit à l'auteur ses études de cas les plus développées. L'ouvrage s'articule ainsi en deux parties, intitulées respectivement 
«Themes in Myth» et "Myths in Tragedy ». La première présente cinq chapitres consacrés à l'étude de thèmes récurrents à travers les différents genres littéraires grecs ; la seconde, qui comprend six chapitres, inverse le focus pour saisir le traitement de quelques thèmes mythiques à l'intérieur d'un genre particulier, le genre tragique, et de quelques unes de ses œuvres singulières.

5 Les deux premiers chapitres s'intéressent à deux thèmes mythiques empruntés au monde naturel, géographique et zoologique : la montagne et le loup. Si la première de ces deux études s'inscrit dans une perspective plus nettement structuraliste, toutes deux s'attachent à montrer comment les représentations mythiques s'élaborent en sélectionnant, en déformant et en accentuant certaines données de l'observation empirique pour construire du sens. Ainsi, les représentations mythiques de la montagne tendent à faire de cet espace marginal et sauvage, potentiellement menaçant, le lieu par excellence de renversements radicaux de la norme culturelle et sociale. De même, l'observation et la réinterprétation biaisée du comportement des loups aboutissent à la construction de modèles pensés pour éclairer la nature de la société humaine et de ses rapports avec l'individu. Ces deux études accordent par ailleurs une place importante au rituel, comme point de convergence entre la réalité vécue et l'imaginaire des représentations mythiques. Le rite de la montée vers le sanctuaire de Zeus Aktaios au sommet du Pélion, qu'accomplissait, à l'époque hellénistique, un groupe choisi de notables arcadiens revêtus d'une peau de bétail ${ }^{2}$, actualise ainsi l'image du pasteur mythique. Le renversement spatial et social accompli par le rite demeure cependant, contrairement à ceux représentés par les mythes, temporaire et symbolique, et n'aboutit finalement qu'à une réaffirmation et à un renforcement de la norme. L'idée de marginalité et d'humanité inversée dont est porteuse la représentation mythique du loup peut être utilisée d'une manière comparable par le rite. C'est ce que montrent deux rituels arcadiens au cours desquels un des participants est supposé se transformer temporairement en loup ${ }^{3}$. R.B. interprète ces rites - d'une manière là encore toute traditionnelle - comme de probables rites de passage à l'âge adulte, où la transformation en loup symboliserait la situation de marginalité et d'inversion des normes à laquelle est soumis le jeune initiant.

6 Le troisième chapitre, qui se tourne pour sa part vers le traitement mythique d'une réalité toute culturelle, les noms propres, prolonge l'exploration thématique entreprise par les deux chapitres précédents dans la mesure où l'exemple développé, celui des noms masculins formés sur l'adjectif melas, «noir », a été associé, depuis les travaux de Pierre Vidal-Naquet, au monde de la montagne et du sauvage. C'est justement cette association que R.B. vient questionner, en montrant qu'un nom ne fait sens que dans le contexte narratif et thématique où il apparait, et qui seul peut activer ou invalider les potentialités sémantiques contenues dans son étymologie. L'examen du contexte énonciatif, présent en mineure dans les deux premiers chapitres surtout soucieux des structures de l'imaginaire, passe désormais au premier plan, permettant de distinguer des cas où la noirceur morale et la dissimulation volontiers associées à la couleur noire semblent bien s'appliquer aux personnages dont le nom est formé sur melas, et d'autres où cette association ne semble pas pertinente.

7 Les quatrième et cinquième chapitres, où se confirme l'importance croissante donnée à l'examen du contexte énonciatif, sont unis par le resserrement du corpus mythologique considéré autour de la légende de Jason et Médée, et par une confrontation entre 
versions discursives et figurations iconographiques, dont l'ouvrage présente des reproductions. Le quatrième chapitre, consacré au personnage de Talos, géant de bronze crétois tué par Médée lors du retour des Argonautes, s'interroge sur la manière dont la figure mythique du géant aide à penser la définition de l'humain. L'ambivalence de Talos, situé au carrefour des catégories du divin, de l'animal et de l'humain, est soulignée par deux thèmes centraux dans le récit d'Apollonios, son unique point faible à la cheville, et l'ichôr - sang immortel des dieux ou épanchement pathologique précurseur de mort - qui s'écoule de sa blessure fatidique. L'effort de contextualisation, là encore, pousse à la prudence interprétative. Si l'utilisation de certains parallèles tirés de la médecine hippocratique (ses emplois du mot ichôr) peut ainsi éclairer la version d'Apollonios, d'autres associations (comme le lien, lui aussi médical, entre cheville et sexualité) ne sont pas pertinentes : la stratégie narrative du poème opère des choix au sein des représentations mentales disponibles. Le cinquième chapitre est pour sa part construit autour de l'opposition structurale mouvement/ immobilité, telle qu'elle se laisse saisir dans le même poème d'Apollonios. R.B. montre que cette dichotomie, récurrente dans la pensée grecque, est utilisée par le poète à une fin sémantique précise: il s'agit de mettre en contraste les deux facettes de son héroöne, en opposant une Médée en perpétuelle agitation, tourmentée par ses passions et en proie au déchirement intérieur, et une Médée magicienne concentrée dans l'effort d'envoûtement, maîtresse d'elle-même et des autres. Cet usage de l'opposition mouvement/immobilité, qui ne se retrouve dans aucune autre version poétique du mythe de Médée pourrait trouver, selon l'A., quelques parallèles - pas tout à fait exacts cependant - dans le langage figuré de la céramique attique et italiote.

La seconde partie de l'ouvrage, centrée sur la relation entre mythe et tragédie, s'ouvre sur trois chapitres de mise au point, destinés à saisir les singularités du genre tragique comme contexte poétique du mythe. Le sixième chapitre cible ainsi ce qui différencie le traitement du mythe dans la tragédie de celui qu'il reçoit dans d'autres formes narratives. La tragédie se caractérise d'abord par le type de sélection qu'elle opère au sein de la matière mythique. Plutôt que la représentation des glorieux exploits des héros, elle tend ainsi à privilégier celle des tensions et dilemmes de l'héroïsme, voire de l'effondrement du héros. La tragédie se singularise en outre par l'espace où elle se déroule: que celui-ci soit compris en termes géographiques, culturels, politiques ou mentaux, il s'agit toujours d'un espace des marges, de l'entre-deux. Elle se distingue enfin par la représentation qu'elle donne des dieux et de leurs relations avec les mortels, centrée sur les aspects conflictuels et sur l'impénétrabilité du divin.

Le septième chapitre approfondit la question du lieu tragique, en l'associant à celle du temps et de l'idéologie pour cerner ce qui fait de la tragédie un genre typiquement athénien. La tragédie, explique R.B., inscrit ces trois éléments dans une dialectique de la distance et de la proximité qui lui permet d'interroger les mythes anciens à la lumière des enjeux présents, mais aussi d'utiliser la matière héroïque pour ébranler, voire mettre en crise l'idéologie athénienne contemporaine. Ce genre poétique, selon l'A., fournit à la cité un outil qui lui permet d'éprouver, avec une pleine maîtrise et sans risque réel, les faiblesses de ses propres structures. Le chapitre 8, enfin, développe une ultime caractéristique commune à l'ensemble du genre tragique, celle qui consiste à accentuer le caractère inexplicable des événements, parfois présentés comme dépourvus de cause apparente : c'est par exemple le cas dans les Bacchantes d'Euripide, pour qui veut déterminer les raisons de la soudaine soumission de Penthée à Dionysos. La diversité souvent contradictoire des interprétations proposées par les spécialistes 
prouve que la recherche même de la cause est vaine, et que l'impossibilité interprétative est elle-même une constituante essentielle du sens, qui pointe vers le mystère et l'incompréhensibilité du divin. L'examen du contexte, montre R.B., est non seulement décisif pour l'élaboration de l'interprétation, mais aussi pour la délimitation de ses ambitions.

10 Les trois derniers chapitres, enfin, étudient le traitement de quelques thèmes et structures mythiques précis à l'intérieur d'œuvres tragiques singulières. Le neuvième chapitre tente ainsi de saisir l'usage sophocléen du thème de la cécité par rapport à celui qu'il reçoit dans les mythes grecs en général. L'examen du matériau mythique, d'Homère aux mythographes d'époque romaine, révèle qu'une faculté de vision supérieure, souvent liée par métaphore à une intellection supérieure, distingue les dieux des hommes, tandis que la cécité apparaît fréquemment comme un châtiment ou comme un défaut compensatoire chez des mortels qui ont transgressé les limites de leur humanité par des facultés de connaissances exceptionnelles. Dans un $\mathrm{v}^{\mathrm{e}}$ siècle athénien passionné par la question de la connaissance humaine et de ses pouvoirs, Sophocle réactive et explicite cette association mythique entre cécité, intellection et limites humaines, tout en l'intégrant à son propre discours tragique, tourné vers les conséquences morales de la finitude humaine.

11 Le chapitre 10, centré sur l'Alceste d'Euripide, explore cinq questions - le motif de la porte, la frontière entre vie et mort, le rôle d'Héraklès, le caractère d'Admète et le ton de la pièce - en insistant sur la nécessité de replacer l'œuvre tragique dans son contexte culturel contemporain, notamment celui de la religion et des rites. Cette approche, qui s'inscrit dans la lignée de celles déployées par des chercheurs comme F. Zeitlin, R. Seaford ou H. Foley, tous attentifs à l'usage des thèmes et symboles rituels dans la tragédie, est particulièrement bien illustrée à travers l'étude de la porte. Cette dernière, qui matérialise l'opposition structurale et structurante, constitutive de la dramaturgie tragique, entre intérieur et extérieur, reçoit dans Alceste une fonction sémantique précise liée à des représentations rituelles impliquant à la fois les rites funéraires et les rites de mariage.

12 Le dernier chapitre enfin, consacré aux Bacchantes d'Euripide, s'intéresse à une opposition structurale mise à l'honneur par les gender studies au cours des dernières décennies, la distinction masculin/féminin et ses éventuels brouillages. Questionnant la féminisation qui affecte plusieurs personnages masculins dans cette tragédie, R. Buxton insiste une nouvelle fois sur la nécessité d'éviter toute interprétation monolithique, et d'opérer des distinctions. En ce qui concerne les dieux d'abord, le brouillage des genres, fonctionnel chez Zeus (qui, de sa cuisse, enfante Dionysos comme une mère), morphologique chez Dionysos, apparaît comme une marque de pouvoir élargi, susceptible de transcender les catégories. Pour le mortel qu'est Penthée en revanche, la féminisation à travers l'adoption d'un travestissement féminin est signe de faiblesse, et de soumission impuissante au pouvoir du dieu qui le manipule. Cette sémantique du brouillage des genres sexuels, propre au système de signification construit par les Bacchantes et à la logique du déroulement dramatique de cette pièce, ne se retrouve, selon l'auteur, dans aucune tragédie, mais rencontre en revanche certains parallèles dans la comédie aristophanienne, signe qu'Euripide, à la fin de sa carrière, aurait cherché à ébranler les frontières génériques.

13 On regrette par endroits que l'A. (peut-être faute d'espace nécessaire) n'aille pas tout à fait au bout de son exigence de contextualisation générique. Le rapprochement entre 
comédie et tragédie que nous venons d'évoquer, hâtivement mentionné à la fin de la dernière étude sans que soient réellement pris en considération les codes et enjeux propres au genre comique, en constitue un bon exemple. La recherche du rire, qui fait un usage abondant des costumes grotesques et des plaisanteries à contenu sexuel, confère au thème du travestissement et de la féminisation un traitement et des effets en réalité bien éloignés de ceux mis en œuvre dans la tragédie des Bacchantes. Une comparaison contrastive précise aurait dès lors pu, sur ce point, s'avérer fructueuse. Le même type de reproche peut être formulé à la lecture du chapitre 5 , où le parallèle entre la poésie d'Apollonios et les représentations iconographiques sur céramique ne fait pas explicitement intervenir les spécificités et contraintes du langage pictural. Il n'en reste pas moins que globalement, la rigueur et la prudence méthodologiques déployées par l'A. tout au long de son enquête, ainsi que la finesse nuancée de ses analyses assurent à l'ouvrage une indiscutable force de conviction.

\section{NOTES}

1. La Grèce de l'imaginaire. Les contextes de la mythologie, Paris, La Découverte, 1996. Édition originale: Imaginary Greece: The Contexts of Mythology, Cambridge, Cambridge University Press, 1994.

2. Héraclide [sous le nom de Dikaiarchos], FHG, t. 2, p. 262.

3. Pausanias, VIII, 2, 6 ; Platon, Rép., 565d ; Pline l'Ancien, Hist. nat. VIII, 81.

\section{AUTEURS}

\section{AJDA LATIFSES}

EHESS - ENS Ulm 